\title{
Performance Analysis of Burst Transmission Mechanism for IEEE 802.11-Based Multi-Hop Wireless LANs
}

Tomoya Tainaka, Hiroyuki Masuyama, Shoji Kasahara, and Yutaka Takahashi ${ }^{1}$

\begin{abstract}
IEEE 802.11-based multihop wireless networks have attracted considerable attention for nextgeneration networks since it can extend the service coverage area without difficulty. However, it is well known that the effective end-to-end throughput (E2ET) degrades due to the overhead caused by the distributed coordination function (DCF) scheme of IEEE 802.11 MAC protocol. To improve this issue, the transmission opportunity (TXOP) mechanism is defined in the IEEE 802.11e standard, with which a wireless node can transmit multiple frames consecutively for a maximum channel occupancy time, called TXOPlimit. This paper considers the performance of the TXOP mechanism for multihop wireless networks. Focusing on a three-node chain topology, we model it as a tandem queueing network with two nodes. The E2ET is derived and the analysis is validated by simulation. Numerical results show that the TXOP mechanism works well for multihop wireless networks. It is also shown that adjusting TXOPlimit is significantly important in order to increase the overall throughput.
\end{abstract}

Keywords: IEEE 802.11e, EDCA, Multihop Wireless LAN, TXOP, Markov Analysis.

\section{INTRODUCTION}

Recently, IEEE 802.11-based multihop wireless networks have attracted much interest for nextgeneration networks [1]. The distributed coordination function (DCF) is a fundamental mechanism of the medium access control (MAC) protocol for IEEE 802.11, employing Carrier Sense Multiple Access with Collision Avoidance (CSMA/CA). The IEEE 802.11 DCF specifies random backoff

\footnotetext{
${ }^{1}$ The authors are with the Graduate School of Informatics, Kyoto University, Kyoto 606-8501, Japan (e-mail: \{tainaka, masuyama\}@sys.i.kyoto-u.ac.jp, \{shoji, takahashi\}@i.kyoto-u.ac.jp).
} 
algorithm, with which each wireless node DCF transmits data in an autonomously distributed manner. It can provide a high-speed wireless connection (e.g. IEEE802.11g provides up to 54 $\mathrm{Mb} / \mathrm{s}$ data rate), however, it is well known that the effective end-to-end throughput (E2ET) degrades due to the overhead caused by the random backoff and the four-way handshake mechanism based on the exchange of Request-To-Send/Clear-To-Send (RTS/CTS) frames. To improve this issue, the transmission opportunity (TXOP) mechanism is defined in IEEE 802.11e, enabling a wireless node to transmit multiple frames consecutively for a maximum channel occupancy time called TXOPlimit [2]. The TXOP mechanism is expected to enhance significantly the capacity in order to accommodate multimedia traffic such as video streaming and Voice over Internet Protocol (VoIP).

It is well known that in multi-hop wireless backhaul networks with homogeneous frame flows, the E2ET of a frame flow degrades significantly with the increase in the number of its transmission hops. Since the TXOP mechanism was developed for single-hop communication, it greatly improves the throughput for single-hop communication. However, it is not clear how the E2ET in multihop wireless networks is improved by the TXOP mechanism. The aim of this paper is to develop an analytical model with which the sensitivity of the TXOPlimit to the E2ET can be quantitatively investigated.

In this paper, we consider how the TXOP mechanism improves the E2ET in a backhaul-type wireless mesh network. Our analysis consists of two steps. First, focusing on a three-node chain topology, we model it as a tandem queueing network with two nodes. With this model, we analyze the joint distribution of the numbers of frames in the two nodes and the server state, deriving the E2ET. Note that in the tandem queueing network model, we simply assume that the time intervals caused by the backoff mechanism are exponentially distributed, depending on which node completes the last frame transmission. In the second step, we analyze the backoff-timer process observed at the completion epoch of a frame transmission. Using the analytical results of the second step, we can determine the parameters of exponential distributions for time intervals caused by the backoff mechanism. We validate the analysis with simulation. 
The rest of the paper is organized as follows. We describe the IEEE 802.11 MAC protocol in Section 3 and present the analytical model in Section 4, which is followed by the analysis of the queueing model in Section 5. We show numerical examples in Section 6 and finally conclude the paper in Section 7.

\section{RELATED WORK}

There is much literature on the performance of TXOP mechanism for multihop wireless networks (see, e.g., $[3,4,5,6]$ ), however, most of the previous studies are based on simulation, and there is little analytical work taking into account the flow-level behavior resulting from the IEEE 802.11 MAC protocol. The pioneering work on performance analysis of the IEEE 802.11 DCF was done by Bianchi [7]. In [7], finite number of nodes and ideal channel conditions were assumed, and frame transmission schemes employed by DCF was considered. The effective throughput under the DCF was analyzed with a discrete time Markov chain.

In terms of single-hop wireless networks, Geyong et al. [8] proposed an analytical model for TXOP mechanism in WLANs under various channel conditions. They modeled the transmission queue of each node by a two-state continuous-time Markov chain which captures the burst transmission mechanism and the wireless channel errors. By using their analytical model, they could obtain the performance metrics including the throughput and buffer overflow probability. Peng et al. [9] also proposed an analytical model to evaluate the performance of TXOP mechanism for single-hop networks. They divided the transmission duration into three different components, successful transmit overhead, collision time overhead and data transmission. They specified the values of these components for two different access modes: basic access and RTS/CTS access, showing how to calculate the total throughput achieved under each access mode. Lee et al. [10] introduced a three dimensional Markov chain model to evaluate the throughput for IEEE 802.11e under saturation condition. Their model captured IEEE 802.11e parameters, and a numerical approach was proposed to obtain the set of parameters to meet the performance requirements. 
In terms of multihop wireless networks, there is little analytical work with regard to the E2ET performance. One of the rationales for the analytical difficulty for multihop wireless networks is that IEEE 802.11 MAC protocol is too complex to model the behavior of multihop frame transmissions. Gao et al. [11] derived the maximum E2ET of multi-hop networks, taking locationdependant neighboring interference into consideration. A strong point of their approach is that the maximum E2ET of any pair of nodes in a multi-hop network can be calculated. However, the impact of parameters such as the offered load and buffer size of a wireless node on the E2ET cannot be investigated. In our three-node tandem network model, on the other hand, location-dependant neighboring interference is not taken into consideration. However, the three-node tandem network model enables us to investigate the sensitivity of the parameters including TXOPlimit to the E2ET.

In our previous work [12], we analyzed the per-flow throughput for IEEE 802.11-based multihop wireless networks. We modeled a three-node chain topology as a tandem queueing network with two nodes, deriving the E2ET. In this paper, we extend the model of [12] to the case in which the TXOP mechanism for IEEE 802.11e is taken into consideration.

\section{BACKOFF AND TXOP MECHANISM}

In this section, we summarize the backoff algorithm and TXOP mechanism, which are modeled and analyzed in the following section. For more details, the readers are referred to [2].

\subsection{Backoff Algorithm of Distributed Coordination Function (DCF)}

In this subsection, we illustrate the backoff algorithm of the IEEE 802.11 DCF. The basic access mechanism of the DCF is CSMA/CA. When a sender node has some data frames to transmit to its receiver node, the sender node first senses the medium before transmission to avoid frame collision. If the medium is idle for a specified time duration called DCF Inter Frame Space (DIFS), RTS/CTS frames are exchanged between the sender and receiver nodes. Then, the sender node transmits a 
data frame to the receiver node. If the data frame is successfully transmitted to the receiver node, the corresponding ACK frame is sent back to the sender node.

After the transmission is completed, the sender node enters the backoff phase. In the backoff algorithm, when a node finds the wireless channel idle, the node sets the backoff timer to a value uniformly distributed within $[0, \mathrm{CW}]$ where $\mathrm{CW}$ is the contention window size. The backoff timer is decreased every slot time while the channel is idle. If the wireless channel is used for the other frame transmission during the backoff period, the node defers decreasing the backoff timer until the channel becomes idle again. When the backoff timer reaches zero, the node starts a frame transmission. $\mathrm{CW}$ is given by $\mathrm{CW}=2^{m} \cdot \mathrm{CW}_{\min }-1$, where $m$ is the number of retransmissions due to frame collision. In a typical setting for IEEE $802.11 \mathrm{~g}$ standard, $\mathrm{CW}_{\min }=16$ and the maximum value of $m$ is 6 . In this case, the initial value of $\mathrm{CW}$ is given by 15 , and $\mathrm{CW}$ is augmented every time collision of frames occurs until reaching 1023. Note that any node is in one of the following four states for the DCF mechanism: "idle state" (the node has no frame to transmit), "busy state" (the wireless channel is used), "inactive state" (the backoff counter of the node decreased), and "transmission state" (the node attempts to transmit).

\subsection{Transmission Opportunity (TXOP) Mechanism}

IEEE 802.11e standard has been issued to introduce quality of service (QoS) support for wireless LANs. The Enhanced Distributed Channel Access (EDCA) is used as the fundamental access mechanism for the MAC layer in IEEE 802.11e. In the EDCA, relative priority service is supported in order to improve the multimedia transmission such as VoIP, and the TXOP is a principal mechanism of the EDCA to guarantee QoS required by real-time applications. To reduce the overhead of the contention time, the TXOP allows multiple consecutive frame exchanges without backoff.

Fig. 1 shows the frame-transmission diagram of three data frames by using the TXOP mechanism. In the TXOP transmission, a sender node can transmit multiple frames within a prespecified time interval called TXOPlimit. After a frame transmission, the next frame is transmitted immedi- 
ately after a successful ACK reception and the Short Inter Frame Space (SIFS). If the sender node can not receive the ACK frame, it terminates the TXOP transmission and sends frames again after another backoff interval. When the TXOPlimit is over, the node goes into backoff phase.

Using TXOP mechanism, data transfer efficiency is improved since the frames included in the same burst share the overhead per transmission opportunity. Hence, the TXOP mechanism provides high throughput performance and low delay for congested networks.

\section{ANALYTICAL MODEL}

We consider a wireless network with three-node chain topology, which consists of Nodes 1 and 2 and the gateway $(\mathrm{GW})$. Node 1 and GW are out of the transmission range but within the sensing range of each other, while each pair of neighboring nodes among the three nodes is within the transmission range. We assume that a single frequency channel is used for frame transmission, which implies that any two nodes cannot use the channel simultaneously. In the wireless network, Flows 1 and 2 are transmitted. Flow 1 is transmitted from Node 1 to GW via Node 2, and Flow 2 is from Node 2 to GW. Nodes 1 and 2 use burst transmission mechanism, and a node having the transmission right sends frames continuously during TXOPlimit.

We model the wireless network as a tandem queueing network with two nodes. (See Fig. 2.) We assume that frames arrive at Node 1 (resp. 2) according to a Poisson process with rate $\lambda_{1}$ (resp. $\lambda_{2}$ ). The system capacity of each node is equal to $K$ (frames). After the transmission right is assigned to Node 1 (resp. 2), Node 1 (resp. 2) sends at most $L_{1}$ (resp. $L_{2}$ ) frames during the TXOPlimit. In each node, the transmission time of $l$ frames is exponentially distributed with rate $\mu^{(l) 2}$. Frames transmitted by Node 1 join the queue of Node 2, while those transmitted by Node

\footnotetext{
${ }^{2}$ This assumption is for analytical and numerical simplicity. We can relax the assumption to allow $l$-frame transmission times to follow a phase-type distribution. It is well known that the family of phase-type distributions is dense in that of all distributions on the set of nonnegative real numbers. However, the phase-type distribution makes the analysis complicated and much computation resources are needed for numerical experiments.
} 
2 leave the network. Either one but not both of the two nodes can transmit a frame when it gets ready for transmission. When the frame transmission of Node 1 (resp. 2) completes, Node 1 or 2 gets ready for frame transmission after a time interval consisting of DIFS and the backoff time. Such interval of Node 1 (resp. 2) is exponentially distributed with rate $\alpha$ (resp. $\beta$ ).

The last assumption (called the inactive-period assumption hereafter) implies that when one of the two nodes completes a frame transmission, each node is forced to be inactive for an exponential time interval. Note that a node in inactive state cannot transmit a frame even when it has the frame in the buffer. If the inactive period of the node is over and the node has no frames to transmit, then the idle period of the node starts. Further when the node is in idle state and a new frame arrives at the node, the node immediately starts the frame transmission.

Let $\operatorname{Pr}(i \rightarrow j)(i, j=1,2)$ denote the probability that the next frame is transmitted by Node $j$ after the frame transmission by Node $i$. Let $\bar{T}$ denote the mean interval between two consecutive completions of frame transmissions in a saturated state, where each node always has at least one frame to transmit. It then follows from the inactive-period assumption that

$$
\bar{T}=1 /(\alpha+\beta)
$$

and for $i=1,2$,

$$
\operatorname{Pr}(i \rightarrow j)= \begin{cases}\alpha /(\alpha+\beta), & j=1 \\ \beta /(\alpha+\beta), & j=2\end{cases}
$$

\section{PERFORMANCE ANALYSIS}

\subsection{Throughput Analysis}

We first define the network states as shown in Table 1 . Let $S(t)(t \geq 0)$ denote the network state at time $t$. Let $N_{1}(t)\left(\right.$ resp. $\left.N_{2}(t)\right)(t \geq 0)$ denote the number of frames in Node 1 (resp. 2) at time $t$. Under the assumptions described in the previous section, the trivariate stochastic process $\left\{\left(N_{1}(t), N_{2}(t), S(t)\right) ; t \geq 0\right\}$ is a continuous-time Markov chain with state space $\mathbb{J}=\mathbb{K} \times \mathbb{K} \times \mathbb{S}$, 
where $\mathbb{K}=\{0,1, \ldots, K\}$ and $\mathbb{S}=\left\{s_{1}, s_{2}, \ldots, s_{8}\right\}$. However, the state space $\mathbb{J}$ includes the states never visited by $\left\{\left(N_{1}(t), N_{2}(t), S(t)\right)\right\}$. We omit those states and construct an irreducible state space $\mathbb{J}^{\prime}$ such that $\mathbb{J}^{\prime}=\left\{\left(n_{1}, n_{2}, s\right) ; n_{1} \in \mathbb{K}, n_{2} \in \mathbb{K}, s \in \mathbb{S}_{n_{1}, n_{2}}\right\}$, where

$$
\mathbb{S}_{n_{1}, n_{2}}= \begin{cases}\left\{s_{4}, s_{5}, s_{6}, s_{7}, s_{8}\right\}, & n_{1}=0, n_{2}=0, \\ \left\{s_{2}, s_{3}, s_{4}, s_{5}, s_{6}\right\}, & n_{1}=0, n_{2} \geq 1, \\ \left\{s_{1}, s_{4}, s_{7}\right\}, & n_{1} \geq 1, n_{2}=0, \\ \left\{s_{1}, s_{2}, s_{3}, s_{4}\right\}, & n_{1} \geq 1, n_{2} \geq 1 .\end{cases}
$$

Note that (i) $\left\{N_{1}(t)\right\}$ increases one by one or decreases by at most $L_{1}$ per transmission; (ii) while $N_{1}(t) \geq 1,\left\{\left(N_{2}(t), S(t)\right)\right\}$ changes the state according to the same law if $\left\{N_{1}(t)\right\}$ increases simultaneously; (iii) while $L_{1}+1 \leq N_{1}(t) \leq K$, the transition of $\left\{\left(N_{2}(t), S(t)\right)\right\}$ is independent of the value of $\left\{N_{1}(t)\right\}$. Therefore the infinitesimal generator $\boldsymbol{Q}$ of $\left\{\left(N_{1}(t), N_{2}(t), S(t)\right)\right\}$ takes the following block-structured form:

$$
\boldsymbol{Q}=\left(\begin{array}{cccccccc}
\boldsymbol{F}_{1} & \boldsymbol{H}_{1} & \boldsymbol{O} & \ldots & \ldots & \ldots & \ldots & \boldsymbol{O} \\
\boldsymbol{G}_{1}^{(1)} & \boldsymbol{F}_{2}^{(1)} & \boldsymbol{H}_{2} & \ddots & & & & \vdots \\
\boldsymbol{G}_{1}^{(2)} & \boldsymbol{O} & \boldsymbol{F}_{2}^{(2)} & \ddots & \ddots & & & \vdots \\
\vdots & \vdots & \ddots & \ddots & \ddots & \ddots & & \vdots \\
\boldsymbol{G}_{1}^{\left(L_{1}\right)} & \boldsymbol{O} & & \boldsymbol{O} & \boldsymbol{F}_{2}^{\left(L_{1}\right)} & \boldsymbol{H}_{2} & \ddots & \vdots \\
\boldsymbol{O} & \boldsymbol{G}_{2} & \boldsymbol{O} & & \ddots & \ddots & \ddots & \boldsymbol{O} \\
\vdots & \ddots & \ddots & \ddots & & \ddots & \boldsymbol{F}_{2}^{\left(L_{1}\right)} & \boldsymbol{H}_{2} \\
\boldsymbol{O} & \ldots & \boldsymbol{O} & \boldsymbol{G}_{2} & \boldsymbol{O} & \ldots & \boldsymbol{O} & \boldsymbol{F}_{2}^{\left(L_{1}\right)}+\boldsymbol{H}_{2}
\end{array}\right)
$$

where the details of $\boldsymbol{F}_{1}, \boldsymbol{F}_{2}^{\left(l_{1}\right)}, \boldsymbol{G}_{1}^{\left(l_{1}\right)}\left(l_{1}=1,2, \ldots, L_{1}\right), \boldsymbol{G}_{2}$ and $\boldsymbol{H}_{i}(i=1,2)$ are found in Appendix A. Because $Q$ is an irreducible finite generator, there exists the unique probability vector $\boldsymbol{\pi}=\left(\pi_{n_{1}, n_{2}, s} ;\left(n_{1}, n_{2}, s\right) \in \mathbb{J}^{\prime}\right)$ such that $\boldsymbol{\pi}>\boldsymbol{0}$ and $\boldsymbol{\pi} \boldsymbol{Q}=\mathbf{0}$. Clearly,

$$
\pi_{n_{1}, n_{2}, s}=\lim _{t \rightarrow \infty} \operatorname{Pr}\left[N_{1}(t)=n_{1}, N_{2}(t)=n_{2}, S(t)=s\right] .
$$


Next we consider the throughputs of Flows 1 and 2, which are denoted by $\theta_{1}$ and $\theta_{2}$, respectively. Let $\theta$ denote the total throughput of Flows 1 and 2 . Let $P_{\text {loss }}^{[2]}$ denote the frame loss probability of Flow 2 at Node 2. We then have $\theta_{1}=\theta-\theta_{2}$ and $\theta_{2}=\bar{U} \lambda_{2}\left(1-P_{\text {loss }}^{[2]}\right)$, where $\bar{U}$ denotes the mean length of a frame. Using PASTA (Poisson Arrivals See Time Averages) (see, e.g., [13]), we can readily obtain

$$
P_{\mathrm{loss}}^{[2]}=\lim _{t \rightarrow \infty} \operatorname{Pr}\left[N_{2}(t)=K\right]=\sum_{\left(n_{1}, K, s\right) \in \mathbb{J}^{\prime}} \pi_{n_{1}, K, s} .
$$

Further since a frame in Flow 2 is transmitted to GW only when $S(t)=s_{2}$, we have

$$
\begin{aligned}
\theta & =\bar{U} \sum_{n_{2}=1}^{K}\left(n_{2} \wedge L_{2}\right) \mu^{\left(n_{2} \wedge L_{2}\right)} \lim _{t \rightarrow \infty} \operatorname{Pr}\left[N_{2}(t)=n_{2}, S(t)=s_{2}\right] \\
& =\bar{U} \sum_{\left(n_{1}, n_{2}, s_{2}\right) \in \mathbb{J}^{\prime}}\left(n_{2} \wedge L_{2}\right) \mu^{\left(n_{2} \wedge L_{2}\right)} \pi_{n_{1}, n_{2}, s_{2}}
\end{aligned}
$$

where $x \wedge y=\min (x, y)$ for any two real numbers $x$ and $y$.

\subsection{Determination of $\alpha$ and $\beta$ by a Markov Model}

In this subsection, we determine parameters $\alpha$ and $\beta$ by modeling the dynamics of the backoff timers of Nodes 1 and 2 as a bivariate Markov chain. For this purpose, we assume $\alpha=\beta$ hereafter. Note that this assumption becomes accurate when the system is under saturated condition. It should be noted that this assumption reduces (1) and (2) to the following:

$$
\begin{aligned}
\bar{T} & =1 /(2 \alpha), \\
\operatorname{Pr}(i \rightarrow j) & =1 / 2, \quad i, j=1,2 .
\end{aligned}
$$

We consider a bivariate process $\left\{\left(Z_{1}(n), Z_{2}(n)\right) ; n=0,1, \ldots\right\}$, where $Z_{i}(n)(i=1,2)$ denotes the backoff-timer value of Node $i$ immediately after the completion of the $n$th successful frame transmission. In what follows, we assume that the arrival rate of frames to each node is so large that the node always has at least one frame to transmit. This assumption shows that the system is in a saturated state. Let $\xi(n)(n=1,2, \ldots)$ denote the number of consecutive collisions that occur after the $n$th successful frame transmission completes. As explained in Section 3.1, each of the three nodes is within the sensing range of the others, and thus consecutive collisions do 
not occur so often. Therefore for the convenience of analysis, we assume that $\xi(n) \leq 1$ for any $n=1,2, \ldots$, i.e., consecutive collisions do not occur during the transmission of a frame, which implies that the maximum value of $\mathrm{CW}$ is limited to 31 . Note here that one of the $Z_{i}(n)$ 's is zero and the other is greater than zero. Thus the state space $\mathbb{Z}$ of $\left\{\left(Z_{1}(n), Z_{2}(n)\right)\right\}$ is given by $\mathbb{Z}=\{(0,1),(0,2), \ldots,(0,31),(1,0),(2,0) \ldots,(31,0)\}$

Let $X_{i}(n)$ denote the backoff timer value that Node $i$ takes for the first time after the completion of the $n$th successful frame transmission. Note that if $Z_{i}(n)>0, X_{i}(n)=Z_{i}(n)$, and otherwise $X_{i}(n)$ takes a value from $\{0,1, \ldots, 15\}$ with equal probability. Let $Y_{i}(n)$ denote the backoff timer value that Node $i$ chooses after a collision occurs, i.e., $X_{1}(n)=X_{2}(n)$. Since $\xi(n) \leq 1$, we assume that $\left(Y_{1}(n), Y_{2}(n)\right)$ take integer values from $\left\{\left(k_{1}, k_{2}\right) ; 0 \leq k_{1}, k_{2} \leq 31, k_{1} \neq k_{2}\right\}$ with equal probability. For simplicity, let $(\imath, \jmath)$ denote $(1,2)$ or $(2,1)$. It then follows that

$$
\begin{aligned}
Z_{\imath}(n+1)= & \mathbb{1}(\xi(n)=0)\left[\mathbb{1}\left(Z_{\imath}(n)=0\right)\left(X_{\imath}(n)-Z_{\jmath}(n)\right)^{+}\right. \\
& \left.+\mathbb{1}\left(Z_{\jmath}(n)=0\right)\left(Z_{\imath}(n)-X_{\jmath}(n)\right)^{+}\right]+\mathbb{1}(\xi(n)=1)\left(Y_{\imath}(n)-Y_{\jmath}(n)\right)^{+},
\end{aligned}
$$

where $\mathbb{1}(\chi)$ denotes the indicator function of event $\chi$ and $(x)^{+}=\max (x, 0)$. Equation (5) shows that the bivariate process $\left\{\left(Z_{1}(n), Z_{2}(n)\right) ; n=0,1, \ldots\right\}$ is a Markov chain with state space $\mathbb{Z}$.

Let $\boldsymbol{P}=\left(P_{i, j} ; i, j \in \mathbb{Z}\right)$ denote the transition probability matrix of the Markov chain $\left\{\left(Z_{1}(n), Z_{2}(n)\right)\right\}$. Arranging the states of $\mathbb{Z}$ in lexicographical order, $\boldsymbol{P}$ takes the following form:

$$
\boldsymbol{P}=\left(\begin{array}{cc}
\boldsymbol{P}_{1} & \boldsymbol{P}_{2} \\
\boldsymbol{P}_{2} & \boldsymbol{P}_{1}
\end{array}\right),
$$

where $\boldsymbol{P}_{i}(i=1,2)$ are given in Appendix B. It is easy to see that $\boldsymbol{P}$ is a finite, irreducible and aperiodic transition matrix. Thus $\boldsymbol{P}$ has the unique stationary probability vector $\varpi=\left(\varpi_{k_{1}, k_{2}} ;\left(k_{1}, k_{2}\right) \in\right.$ $\mathbb{Z})$ such that $\varpi_{k_{1}, k_{2}}=\lim _{n \rightarrow \infty} \operatorname{Pr}\left[Z_{1}(n)=k_{1}, Z_{2}(n)=k_{2}\right]$. Since $\boldsymbol{P}$ is a $62 \times 62$ stochastic matrix, its stationary probability vector $\varpi$ can be easily obtained by solving a system of equations $\varpi \boldsymbol{P}=\varpi$ and $\sum_{\left(k_{1}, k_{2}\right) \in \mathbb{Z}} \varpi_{k_{1}, k_{2}}=1$.

We now define $\widetilde{\varpi}_{0, k}$ and $\widetilde{\varpi}_{k, 0}(k=1,2, \ldots, 31)$ as

$$
\widetilde{\varpi}_{0, k}=\operatorname{Pr}\left[Z_{2}(n)=k \mid Z_{1}(n)=0\right], \quad \widetilde{\varpi}_{k, 0}=\operatorname{Pr}\left[Z_{1}(n)=k \mid Z_{2}(n)=0\right],
$$


respectively. We then have $\widetilde{\varpi}_{0, k}=\varpi_{0, k} / \sum_{m=1}^{31} \varpi_{0, m}$ and $\widetilde{\varpi}_{k, 0}=\varpi_{k, 0} / \sum_{m=1}^{31} \varpi_{m, 0}$, respectively.

Let $T(n)$ denote the time interval from the completion epoch of the $n$th successful frame transmission to the beginning of the $(n+1)$ st successful frame transmission. We then have

$$
\begin{aligned}
T(n)=\operatorname{DIFS} & +\mathbb{1}(\xi(n)=0) \cdot \tau\left(X_{\imath}(n) \wedge X_{\jmath}(n)\right)+\mathbb{1}(\xi(n)=1) \cdot\left[T_{c}+\tau\left\{X_{\imath}(n)+\left(Y_{\imath}(n) \wedge Y_{\jmath}(n)\right)\right\}\right] \\
=\mathrm{DIFS} & +\mathbb{1}\left(X_{\imath}(n) \neq X_{\jmath}(n)\right) \cdot \tau\left(X_{\imath}(n) \wedge X_{\jmath}(n)\right) \\
& \quad+\mathbb{1}\left(X_{\imath}(n)=X_{\jmath}(n)\right) \cdot\left[T_{c}+\tau\left\{X_{\imath}(n)+\left(Y_{\imath}(n) \wedge Y_{\jmath}(n)\right)\right\}\right],
\end{aligned}
$$

where $\tau$ is the slot size and $T_{c}$ is the time wasted in collision. Since $\bar{T}=\lim _{n \rightarrow \infty} \mathrm{E}[T(n)]$, it follows from (4) and (6) that

$$
\begin{aligned}
\bar{T}=\operatorname{DIFS}+\lim _{n \rightarrow \infty} \mathrm{E}\left[\mathbb{1}\left(X_{1}(n) \neq Z_{2}(n)\right) \cdot \tau\left(X_{1}(n) \wedge Z_{2}(n)\right) \mid Z_{1}(n)=0\right] \\
+\lim _{n \rightarrow \infty} \mathrm{E}\left[\mathbb{1}\left(X_{1}(n)=Z_{2}(n)\right) \cdot\left[T_{c}+\tau\left\{X_{1}(n)+\left(Y_{1}(n) \wedge Y_{2}(n)\right)\right\}\right] \mid Z_{1}(n)=0\right] \\
=\text { DIFS }+\tau\left\{\sum_{k=1}^{15} \widetilde{\varpi}_{0, k}\left(\sum_{m=0}^{k-1} \frac{m}{16}+\sum_{m=k+1}^{15} \frac{k}{16}\right)+\sum_{k=16}^{31} \widetilde{\varpi}_{0, k} \sum_{m=0}^{15} \frac{m}{16}\right\} \\
+\sum_{k=1}^{15} \widetilde{\varpi}_{0, k} \cdot \frac{1}{16}\left[T_{c}+\tau\left(k+\sum_{m=0}^{31} m \cdot \frac{2(31-m)}{32 \cdot 31}\right)\right] \\
=\operatorname{DIFS}+\tau\left\{\sum_{k=1}^{15} \widetilde{\varpi}_{0, k} \frac{-k^{2}+29 k}{32}+\sum_{k=16}^{31} \widetilde{\varpi}_{0, k} \frac{15}{2}+\sum_{k=1}^{15} \widetilde{\varpi}_{0, k} \frac{k+10}{16}\right\}+\sum_{k=1}^{15} \widetilde{\varpi}_{0, k} \frac{T_{c}}{16} .
\end{aligned}
$$

As a result, we can determine $\alpha(=\beta)$ by (3) and (7).

\section{NUMERICAL EXAMPLES}

In this section, we show some numerical results calculated from the analysis in the previous section. In Table 2, we show basic parameter values used in numerical experiments. The parameters are cited in the widely used IEEE $802.11 \mathrm{~g}$ standard. We set the system capacity $K=100$ [frames] and the mean frame length $\bar{U}=1500$ [bytes]. With this $\bar{U}$, the mean frame-transmission time is given by $1 / \mu^{(l)}=270 l+469[\mu \mathrm{s}]$. The time wasted in collision $T_{c}$ is set to zero because $T_{c}$ does not affect the performance significantly under the assumption in which frame collision occurs at most once during a frame transmission. 
We use the following fairness index [14] as a measure of the per-flow throughput fairness in case where the offered loads are same.

$$
\text { Fairness Index }=1-\sum_{i=1}^{2} \frac{\left|x_{i}-\bar{x}\right|}{2 \bar{x}}
$$

where $x_{i}$ is the E2ET of Flow $i(i=1,2)$, and $\bar{x}$ is the average E2ET achieved by two flows. The fairness index lies in the range from 0 to 1 , and the value close to 1 indicates high per-flow fairness.

In order to validate the analytical model, we conducted simulation experiments. The simulation program was developed with $\mathrm{C}$ language. In simulation, the frame arrival process at Node 1 (resp. 2) is assumed to be a Poisson process with rate $\lambda_{1}$ (resp. $\lambda_{2}$ ) and the transmission time of $l$ frames at Nodes 1 and 2 is constant with rate $\mu^{(l)}$. Note that the transmission time is assumed to follow exponential distribution in our analysis. The main difference between analysis and simulation is the model of the backoff-timer process. In the analysis, we assumed the saturated condition and the backoff interval to be exponentially distributed, while the backoff procedure presented in subsection 3.1 was performed in simulation. In our simulation experiment, the simulated time was set to 100 seconds. We collected simulation data after the warmup period of 10 seconds in order to remove the initial transient effect on simulation results. The simulation was repeated 30 times for each parameter set and we calculate a 95\% confidence interval.

\subsection{Analytical Model Validation}

Fig. 3 shows the analytical and simulation results of the E2ETs of Flow 1 and Flow 2, respectively, against the offered load in case of $L_{1}=L_{2}=1,3$, and 10. Here, the offered load is given by the mean frame arrival rate multiplied by the mean frame size, and its unit is $\mathrm{Mb} / \mathrm{s}$. The offered load of Flow 1 is the same as that of Flow 2. For simulation results, we show the $95 \%$ confidence intervals with vertical bars.

In Fig. 3, the analytical results agree fairly well with the simulation ones when the offered load is small. When the offered load is large, however, we observe a discrepancy between analysis and simulation. For example, in case of $L_{1}=L_{2}=3$, the percent error of Flow 1 is $0.12 \%$ at $6 \mathrm{Mb} / \mathrm{s}$ 
and $8.08 \%$ at $10 \mathrm{Mb} / \mathrm{s}$, while that of Flow 2 is $0.46 \%$ at $6 \mathrm{Mb} / \mathrm{s}$ and $6.17 \%$ at $10 \mathrm{Mb} / \mathrm{s}$. One of the reasons is that consecutive time intervals due to backoff are strongly correlated in the simulation model. This correlation nature is not taken into account in the analytical model. Note that the saturated condition is assumed only for the backoff-timer process. This may affect the analytical result when the saturated condition does not hold, i.e., the offered load is small. In Fig. 3, however, discrepancy between analysis and simulation is significantly small for a small offered load. When the offered load is small, frame-loss events hardly occur. This makes the impact of the backofftimer process on the E2ET small, resulting in a good agreement between analysis and simulation.

It is also observed from Fig. 3 that the E2ETs of Flows 1 and 2 are the same and linearly increase when the offered load is small. This is because frame loss rarely occurs and hence both the flows achieve the same throughput. When the offered load is large, the E2ET of Flow 1 is smaller than that of Flow 2. Note that frames of Flow 1 suffer from loss at Nodes 1 and 2, while frame loss for Flow 2 occurs only at Node 2. Therefore, frames of Flow 1 are more likely to be dropped than those of Flow 2.

Fig. 4 represents the overall throughput against the offered load in case of $L_{1}=L_{2}=1,3$, and 10. The overall throughput is given by the sum of the E2ETs of Flows 1 and 2. Here, the offered loads of Flows 1 and 2 are the same. A remarkable point here is that the analytical results significantly agree with the simulation results. We also observe from Fig. 4 that the overall throughput grows linearly first, then decreases gradually and finally remains constant with the increase in the offered load. When the offered loads of Flows 1 and 2 increase, Node 2 which is close to GW is more likely to be in congestion. The behavior of queues in the network is classified into the following three situations [15]: (1) Both the queues of Nodes 1 and 2 are not saturated. (2) Nodes 1 is not saturated, while Nodes 2 is saturated. In this case, frames sent to Nodes 2 are likely to be dropped. (3) Both Nodes 1 and 2 are saturated.

For example, in case of $L_{1}=L_{2}=1$, the first situation occurs when the offered load is in the range from $0 \mathrm{Mb} / \mathrm{s}$ to $3 \mathrm{Mb} / \mathrm{s}$. The second situation emerges when the offered load is $3 \mathrm{Mb} / \mathrm{s}$ to 5 
$\mathrm{Mb} / \mathrm{s}$. The last situation arises when the offered load is greater than $5 \mathrm{Mb} / \mathrm{s}$. In the first situation, the overall throughput grows linearly because frame loss rarely occurs. In the second situation, Node 2 sends frames more frequently than Node 1 because the idle state probability of Node 1 is greater than that of Node 2. In the third situation, on the other hand, the average number of transmissions per unit time for Node 1 is equal to that for Node 2. Note that the overall transmission frequency of the wireless channel in the second situation is the same as the third situation. This implies that the overall throughput of the second situation is larger than that of the third situation, because the overall throughput depends entirely upon the number of Node 2's transmissions.

Fig. 5 represents fairness indices against the offered load in case of $L_{1}=L_{2}=1,3$, and 10 . We observe high per-flow fairness at a small offered load. When the offered load increases, however, Flow 1 suffers from throughput unfairness due to a high frame loss probability at Node 2 . It is also observed that the fairness index is improved with the increase in TXOPlimit. This is simply because the channel capacity is increased by the overhead reduction of the TXOP mechanism. It is also noticeable from Figs. 3 to 5 that setting $L_{1}=L_{2}=10$ gives the largest E2ETs of both flows, keeping high throughput fairness.

\subsection{Throughput Performance}

We next discuss how the value of the TXOPlimit affects the throughput performance. Fig. 6 represents E2ETs of Flows 1 and 2 against TXOPlimit $L_{1}$ and $L_{2}$. In this figure, the offered loads of both flows are the same and equal to $8 \mathrm{Mb} / \mathrm{s}$ and $12 \mathrm{Mb} / \mathrm{s}$. It is observed from Fig. 6 that when TXOPlimit is small, the E2ETs of Flows 1 and 2 for all the cases rapidly increase. From the fact, we can improve the throughput performance for multihop wireless networks to use TXOP mechanism. However, for $L_{1}, L_{2}>20$, all the E2ETs in the figure remain almost constant. Using TXOP mechanism for multihop wireless networks has both positive and negative effects. The positive effect is the reduction of the overhead caused by the backoff algorithm and by the exchange of control frames. This enables wireless nodes to send data frames efficiently. 
On the other hand, the negative effect is that the node having the transmission right is likely to use the network channel during a large period of time. Occasionally, this channel occupation leads to deteriorate the performance in multihop wireless networks. In our model, if Node 1 occupies the network channel for a long term and sends lots of frames at a time, the Node 2's queue is overflowed, degrading the throughput performance significantly.

Fig. 7 (resp. Fig. 8) represents the E2ET of Flow 1 (resp. Flow 2) against TXOPlimit $L_{1}$. In these figures, we set $L_{2}=1,5$, and 30, and the offered load of two flows are the same and equal to $10 \mathrm{Mb} / \mathrm{s}$. In Fig. 7, the maximum E2ET for $L_{2}=30$ is nearly three times greater than the E2ET without burst transmissions $\left(L_{1}=1\right)$. On the contrary, E2ETs of the other cases are almost insensitive to the TXOPlimit $L_{1}$. Node 2 is more likely to be saturated when Node 2's TXOPlimit $L_{2}$ is small. Hence, the number of the Flow 1's frames which can enter the Node 2's queue does not depend on the Node 1's TXOPlimit value. In Fig. 8, the maximum E2ET is achieved at $L_{1}=1$ for all the cases. This implies that using TXOP mechanism at Node 1 is ineffective for improving the E2ET of Flow 2. This is simply because Node 1 sends only Flow 1's frames and therefore the channel occupation time for Flow 2's frames relatively decreases for a large Node 1's TXOPlimit.

Finally, we consider the impact of $L_{2}$ on the E2ET. Fig. 9 (resp. Fig. 10) shows E2ETs of Flow 1 (resp. Flow 2) against TXOPlimit $L_{2}$. We set $L_{1}=1,5$, and 30, and the offered load of two flows are equal to $10 \mathrm{Mb} / \mathrm{s}$. In Fig. 9, when the Node 2's TXOPlimit $L_{2}$ increases, the Flow 1's E2ET becomes large and then remains constant for all $L_{1}$ values. Fig. 10 shows the same tendency as Fig. 9. These results show that TXOP mechanism works well for the Node 2's transmission. Note that we need not to set a large TXOPlimit in order to achieve the best performance.

Note that in Figs. 7 to 10, the E2ET doesn't change significantly when the TXOPlimit values of the two nodes are greatly different. This suggests that under a homogeneous offered load condition for this backhaul-type wireless network, setting the TXOPlimits of nodes to the same value is effective to achieve high throughput fairness. Reminding the results of previous subsection, $L_{1}=$ $L_{2}=10$ is the recommended setting for TXOPlimit for this numerical scenario. 


\section{CONCLUSION}

In this paper, we have considered the performance of the TXOP mechanism for multihop wireless networks. We have analyzed the E2ET in a backhaul-type wireless mesh network using a continuous-time Markov chain. We have derived the E2ET and validated the analysis with simulation. From numerical results, we have confirmed that the analytical results agree well with the simulation. Numerical results also showed that a large TXOPlimit causes the degradation of the E2ET, and that under a homogeneous offered load condition, setting the TXOPlimits of nodes to the same value is effective to achieve high throughput fairness. Note that the analytical model in the paper consists of two parts: a three-node network model and a backoff-timer process model. Here, the frame-level queueing behavior is characterized without considering the detailed behavior of the backoff timer. This analytical approach was originally developed by Bianchi [7]. From the modeling point of view, our study verified the effectiveness of the analytical framework developed by [7]. This suggests that two-model based approach is significantly useful for performance analysis of IEEE 802.11-based network systems in which frame-level behavior is mainly concerned.

\section{APPENDIX A: INFINITESIMAL GENERATOR $Q$}

For the sake of saving space, let $\lambda=\lambda_{1}+\lambda_{2}$. Then $\boldsymbol{F}_{1}, \boldsymbol{F}_{2}^{\left(l_{1}\right)}, \boldsymbol{G}_{1}^{\left(l_{1}\right)}\left(l_{1}=1,2, \ldots, L_{1}\right), \boldsymbol{G}_{2}$ and $\boldsymbol{H}_{i}(i=1,2)$ can be written as follows:

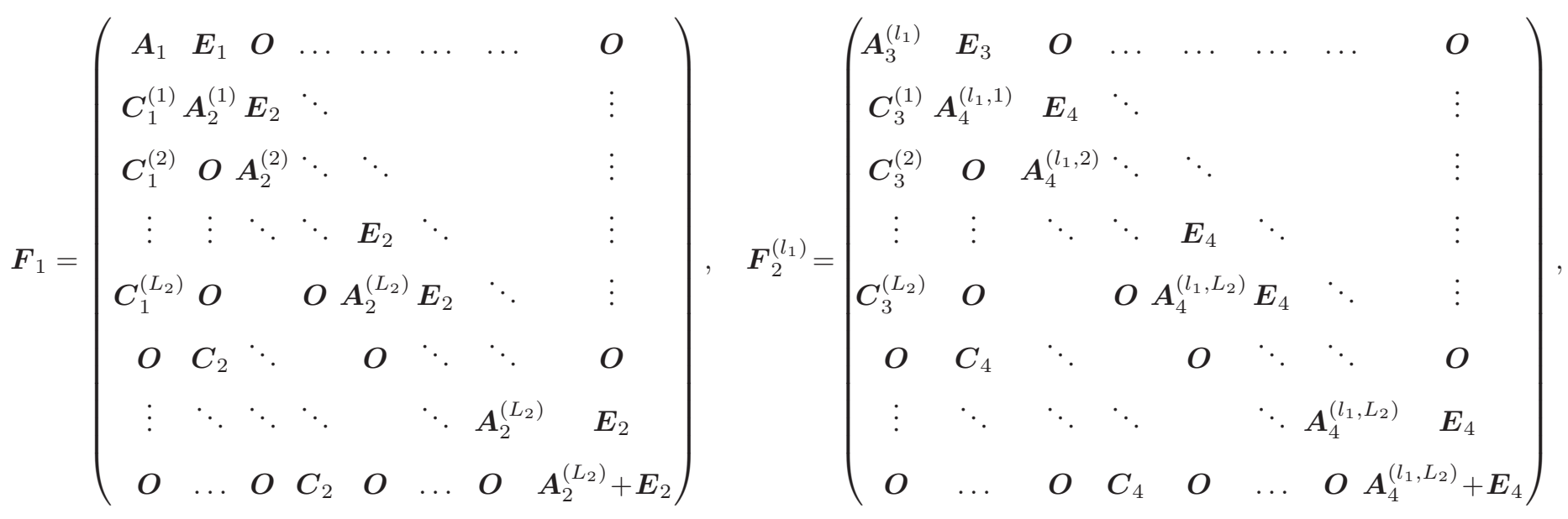




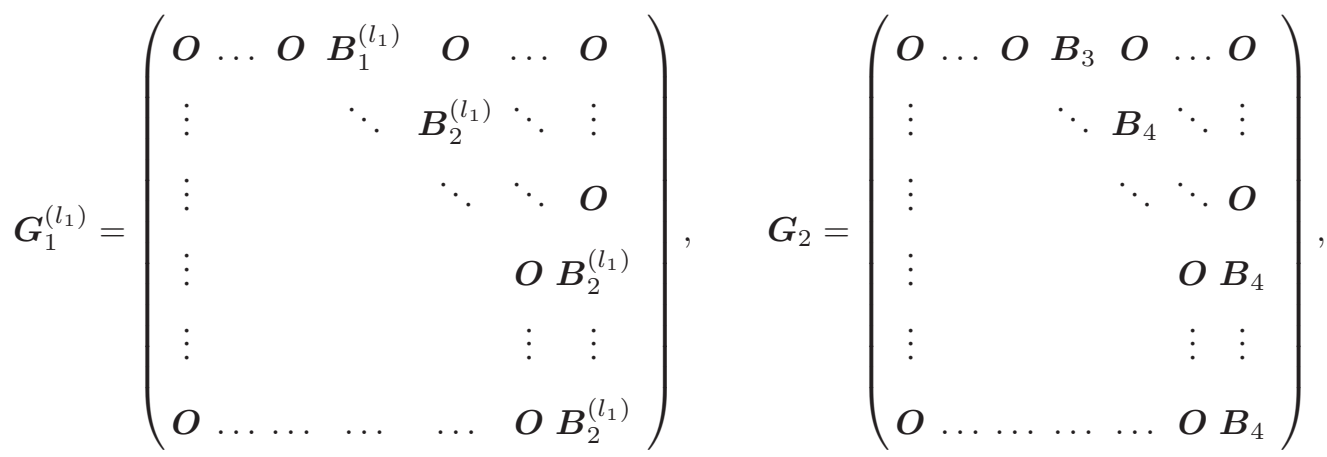

$$
\begin{aligned}
& H_{1}=\left(\begin{array}{cccccc}
D_{1} & O & O & \ldots & O & O \\
O & D_{2} & O & \ddots & \vdots & \vdots \\
O & O & D_{2} & \ddots & O & O \\
O & O & O & \ddots & O & O \\
\vdots & \vdots & \ddots & \ddots & D_{2} & O \\
O & O & O & \ldots & O & D_{2}
\end{array}\right), \quad H_{2}=\left(\begin{array}{cccccc}
D_{3} & O & O & \ldots & O & O \\
O & D_{4} & O & \ddots & \vdots & \vdots \\
O & O & D_{4} & \ddots & O & O \\
O & O & O & \ddots & O & O \\
\vdots & \vdots & \ddots & \ddots & D_{4} & O \\
O & O & O & \ldots & O & D_{4}
\end{array}\right),
\end{aligned}
$$

where $\boldsymbol{A}_{1}, \boldsymbol{A}_{2}^{\left(l_{2}\right)}, \boldsymbol{A}_{3}^{\left(l_{1}\right)}, \boldsymbol{A}_{4}^{\left(l_{1}, l_{2}\right)}, \boldsymbol{B}_{1}^{\left(l_{1}\right)}, \boldsymbol{B}_{2}^{\left(l_{1}\right)}, \boldsymbol{B}_{3}, \boldsymbol{B}_{4}, \boldsymbol{C}_{1}^{\left(l_{2}\right)}, \boldsymbol{C}_{2}, \boldsymbol{C}_{3}^{\left(l_{2}\right)}, \boldsymbol{C}_{4}\left(l_{1}=1,2, \ldots, L_{1}\right.$, $\left.l_{2}=1,2, \ldots, L_{2}\right), \boldsymbol{D}_{i}$, and $\boldsymbol{E}_{i}(i=1,2,3,4)$ are defined in the following way.

$$
\begin{aligned}
& \boldsymbol{A}_{1}=\left(\begin{array}{ccccc}
-\alpha-\beta-\lambda & 0 & \beta & \alpha & 0 \\
0 & -\beta-\lambda & 0 & 0 & \beta \\
0 & 0 & -\alpha-\lambda & 0 & \alpha \\
0 & 0 & 0 & -\beta-\lambda & \beta \\
0 & 0 & 0 & 0 & -\lambda
\end{array}\right), \quad \boldsymbol{A}_{2}^{\left(l_{2}\right)}=\left(\begin{array}{rrrrr}
-\mu^{\left(l_{2}\right)}-\lambda & 0 & 0 & 0 & 0 \\
\beta & -\alpha-\beta-\lambda & 0 & \alpha & 0 \\
\alpha & 0 & -\alpha-\beta-\lambda & 0 & \beta \\
\beta & 0 & 0 & -\beta-\lambda & 0 \\
\alpha & 0 & 0 & 0-\alpha-\lambda
\end{array}\right), \\
& \boldsymbol{A}_{3}^{\left(l_{1}\right)}=\left(\begin{array}{ccc}
-\mu^{\left(l_{1}\right)}-\lambda & 0 & 0 \\
\beta & -\alpha-\beta-\lambda & \alpha \\
\beta & 0 & -\beta-\lambda
\end{array}\right), \quad \boldsymbol{A}_{4}^{\left(l_{1}, l_{2}\right)}=\left(\begin{array}{rrrr}
-\mu^{\left(l_{1}\right)}-\lambda & 0 & 0 & 0 \\
0 & -\mu^{\left(l_{2}\right)}-\lambda & 0 & 0 \\
\alpha & \beta & -\alpha-\beta-\lambda & 0 \\
\beta & \alpha & 0 & -\alpha-\beta-\lambda
\end{array}\right),
\end{aligned}
$$

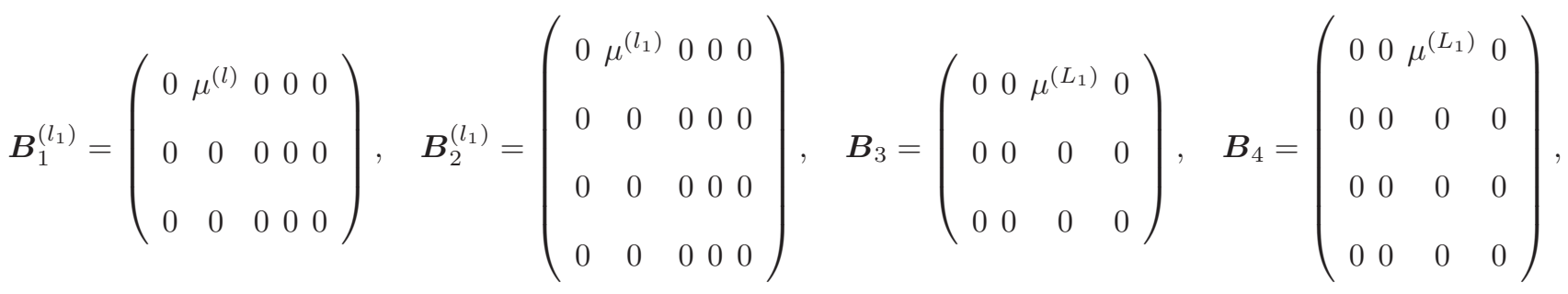




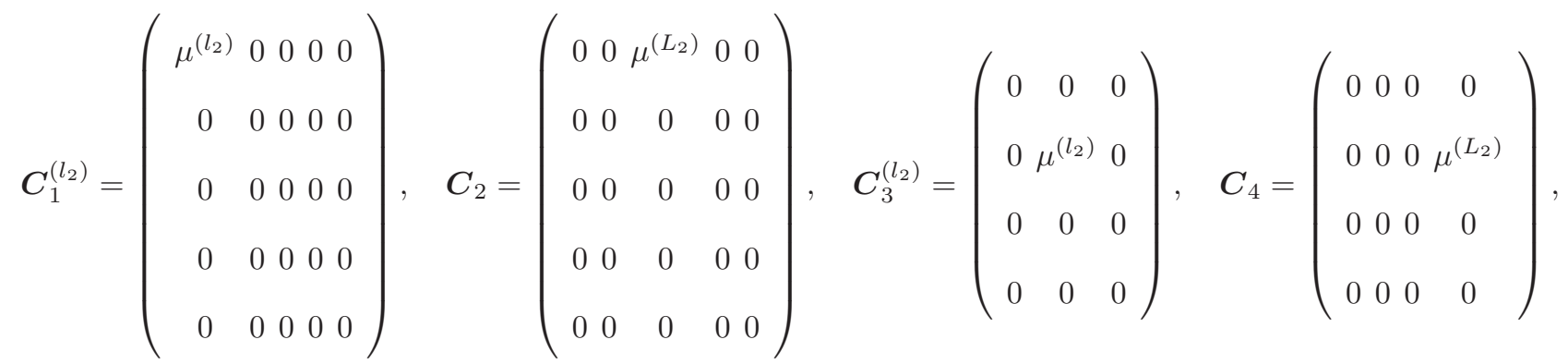

$$
\begin{aligned}
& \boldsymbol{D}_{1}=\left(\begin{array}{ccc}
0 & \lambda_{1} & 0 \\
\lambda_{1} & 0 & 0 \\
\lambda_{1} & 0 & 0 \\
0 & 0 & \lambda_{1} \\
\lambda_{1} & 0 & 0
\end{array}\right), \quad \boldsymbol{D}_{2}=\left(\begin{array}{cccc}
0 & \lambda_{1} & 0 & 0 \\
0 & 0 & \lambda_{1} & 0 \\
0 & 0 & 0 & \lambda_{1} \\
\lambda_{1} & 0 & 0 & 0 \\
\lambda_{1} & 0 & 0 & 0
\end{array}\right), \boldsymbol{D}_{3}=\left(\begin{array}{ccc}
\lambda_{1} & 0 & 0 \\
0 & \lambda_{1} & 0 \\
0 & 0 & \lambda_{1}
\end{array}\right), \quad \boldsymbol{D}_{4}=\left(\begin{array}{cccc}
\lambda_{1} & 0 & 0 & 0 \\
0 & \lambda_{1} & 0 & 0 \\
0 & 0 & \lambda_{1} & 0 \\
0 & 0 & 0 & \lambda_{1}
\end{array}\right), \\
& \boldsymbol{E}_{1}=\left(\begin{array}{ccccc}
0 & 0 & \lambda_{2} & 0 & 0 \\
0 & 0 & 0 & \lambda_{2} & 0 \\
0 & 0 & 0 & 0 & \lambda_{2} \\
\lambda_{2} & 0 & 0 & 0 & 0 \\
\lambda_{2} & 0 & 0 & 0 & 0
\end{array}\right), \quad \boldsymbol{E}_{2}=\left(\begin{array}{ccccc}
\lambda_{2} & 0 & 0 & 0 & 0 \\
0 & \lambda_{2} & 0 & 0 & 0 \\
0 & 0 & \lambda_{2} & 0 & 0 \\
0 & 0 & 0 & \lambda_{2} & 0 \\
0 & 0 & 0 & 0 & \lambda_{2}
\end{array}\right), \quad \boldsymbol{E}_{3}=\left(\begin{array}{cccc}
\lambda_{2} & 0 & 0 & 0 \\
0 & 0 & 0 & \lambda_{2} \\
0 & \lambda_{2} & 0 & 0
\end{array}\right), \quad \boldsymbol{E}_{4}=\left(\begin{array}{cccc}
\lambda_{2} & 0 & 0 & 0 \\
0 & \lambda_{2} & 0 & 0 \\
0 & \lambda_{2} & 0 \\
0 & 0 & \lambda_{2}
\end{array}\right) .
\end{aligned}
$$

\section{Appendix B: Elements of transition probability matrix $P$}

The block matrices $\boldsymbol{P}_{i}$ 's $(i, j=1,2)$ of $\boldsymbol{P}$ are $32 \times 32$ matrices given by
$(0,1)(0,2) \cdots \cdots$

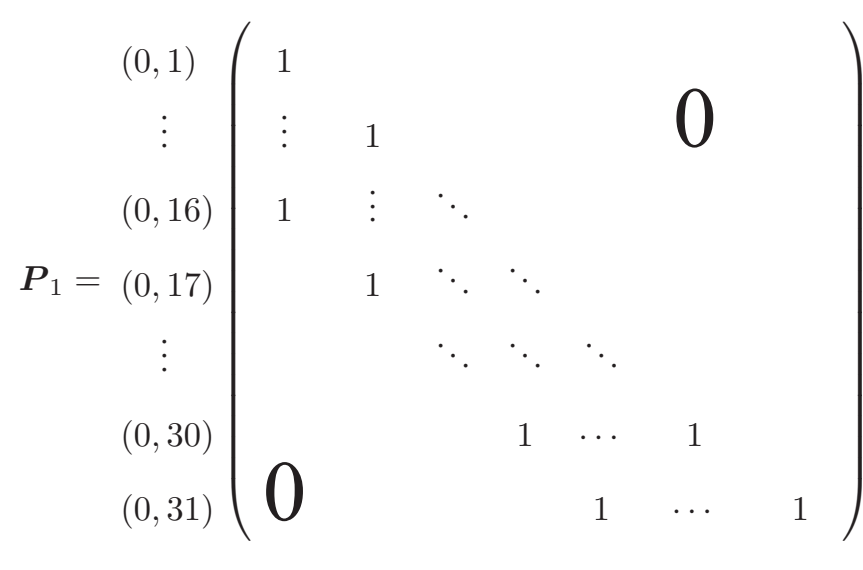

$(0,1)(0,2) \cdots(0,30)(0,31)$

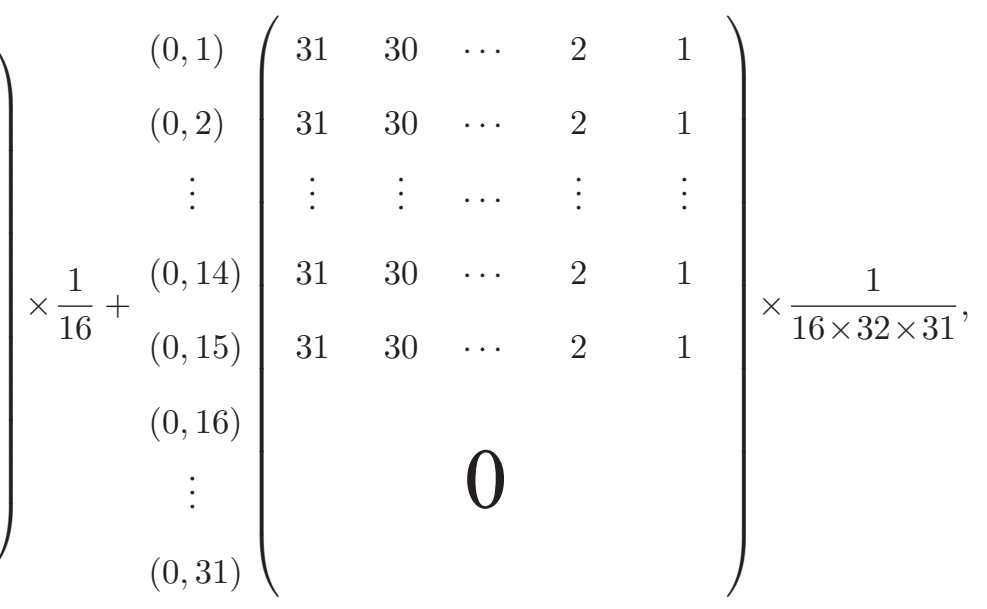




$$
\begin{gathered}
(0,1) \\
\vdots \\
\left.P_{2}=\begin{array}{c}
(1,0) \\
(0,14) \\
(0,15) \\
\vdots \\
(0,31)
\end{array}\right) \\
\end{gathered}
$$

\section{References}

[1] I. F. Akyildiz, X. Wang and W. Wang, "Wireless mesh networks: A survey," Comput. Netw., vol. 47, no. 4, pp. 445-487, 2005.

[2] IEEE Standard 802.11: Wireless LAN medium access control (MAC) and physical layer (PHY) specifications: Medium access control (MAC) quality of service (QoS) enhancements, IEEE 802.11e/D13.0, 2005.

[3] S. Choi, J. Prado, S. Mangold, and S. Shankar, "IEEE 802.11e contention-based channel access (EDCF) performance evaluation,” in Proc. IEEE ICC'03, 2003, pp.1151-1156.

[4] A. Grilo and M. Nunes, "Performance evaluation of IEEE 802.11e," in Proc. IEEE PIMRC'02, Sep. 2002, pp. 511-517.

[5] F. Keceli, I. Inan and E. Ayanoglu, "Fair access provisioning through contention parameter adaptation in the IEEE 802.11e infrastructure basic service set," Arxiv preprint, arXiv:0806.1093, 2008.

[6] T. Suzuki, A. Noguchi and S. Tasaka, "Effect of TXOP-bursting and transmission error on application-level and user-level QoS in audio-video transmission with IEEE 802.11e EDCA," in Proc. IEEE PIMRC'06, Sep. 2006, pp. 1-7. 
[7] G. Bianchi, "Performance analysis of the IEEE 802.11 distributed coordination function," IEEE J. Sel. Areas in Commun., vol. 18, no. 3, pp. 535-48, 2000.

[8] G. Min, J Hu, W. Jia and M. E. Woodward, "Performance analysis of the TXOP scheme in IEEE 802.11e WLANs with bursty error channels," in Proc. IEEE WCNC'09, 2009, pp. 1185-1190.

[9] F. Peng, H. M. Alnuweiri and V. C. M. Leung, "Analysis of burst transmission in IEEE 802.11e wireless LANs," in Proc. IEEE ICC'06, Jun. 2006, vol. 2, pp. 535-539.

[10] Y. Lee, K. S. Lee and J. M. Jang, "Saturation throughput analysis of IEEE 802.11e EDCA," Lecture Notes in Computer Science, no. 4682, pp. 1223-1232, 2007.

[11] Y. Gao, D. M. Chiu, J. C. S. Lui, "Determining the end-to-end throughput capacity in multihop networks: methodology and applications," in Proc. the joint international conference on measurement and modeling of computer systems, Jun. 2006, pp. 39-50.

[12] T. Tainaka, H. Masuyama, S, Kasahara, Y. Takahashi , "A Markovian approach to per-flow throughput unfairness in IEEE 802.11 multihop wireless networks," Journal of Industrial and Management Optimization, vol. 5, no. 3, pp. 493-510, 2009.

[13] R. W. Wolf, "Poisson arrivals see time averages," Operations Research, vol. 30, pp. 223-231, 1982.

[14] D. Vardalis, “On the efficiency and fairness of TCP over wired/wireless networks," Master Thesis, State University of New York at Stony Brook, 2001.

[15] P. T. Giang and K. Nakagawa, "Improvement of fairness by PCRQ scheduling in multihop wireless adHoc networks," in Proc. Asia-Pacific Symposium on Queueing Theory and Network Applications, 2007, pp. 339-348. 


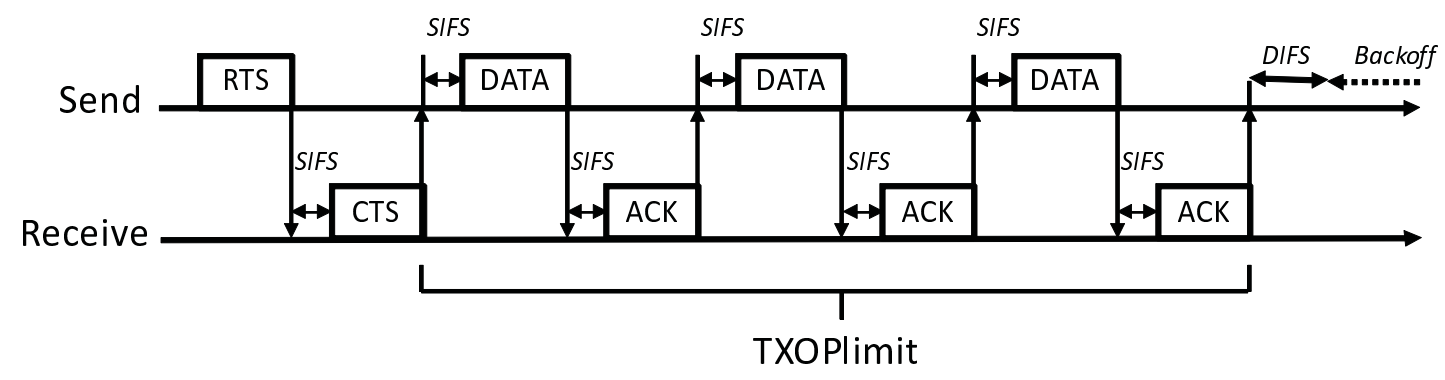

Figure 1: Transmission Opportunity (TXOP) mechanism.

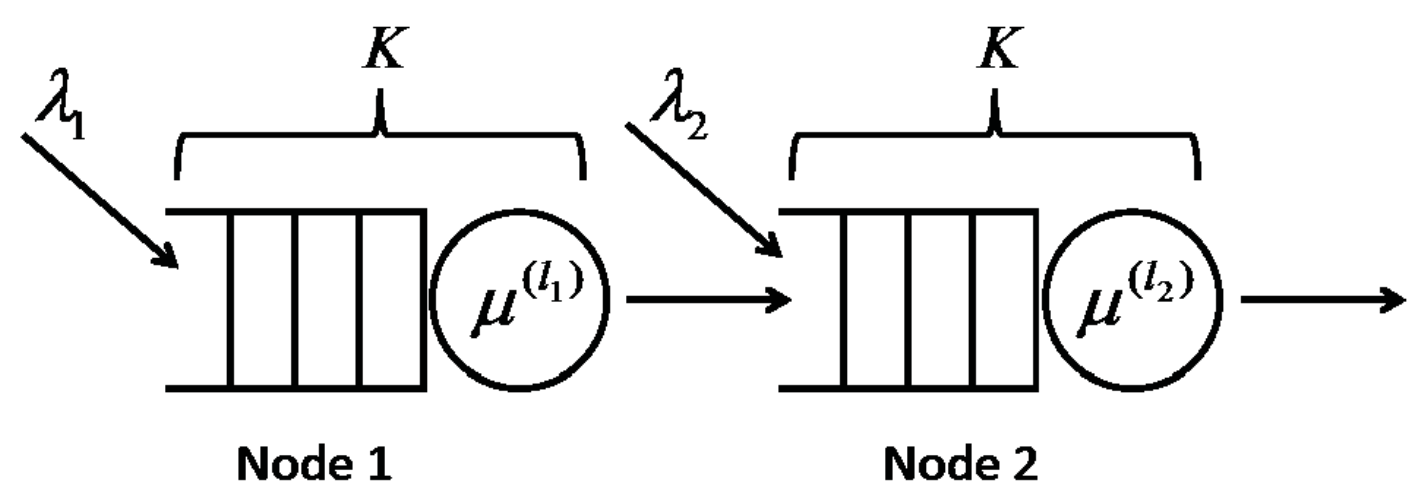

Figure 2: Queueing model. 
Table 1: Definition of network state.

\begin{tabular}{|c|l|}
\hline State & Meaning \\
\hline$s_{1}$ & The service is in progress at Node 1. \\
$s_{2}$ & The service is in progress at Node 2. \\
$s_{3}$ & Nodes 1 and 2 are inactive after a frame transmission by Node 1. \\
$s_{4}$ & Nodes 1 and 2 are inactive after a frame transmission by Node 2. \\
$s_{5}$ & Node 1 is idle and Node 2 is inactive after a frame transmission by Node 1. \\
$s_{6}$ & Node 1 is idle and Node 2 is inactive after a frame transmission by Node 2. \\
$s_{7}$ & Node 1 is inactive and Node 2 is idle after a frame transmission by Node 2. \\
$s_{8}$ & Nodes 1 and 2 are idle.
\end{tabular}

Table 2: IEEE $802.11 \mathrm{~g}$ standard.

\begin{tabular}{|l|c|}
\hline Frame transmission rate & $54[\mathrm{Mb} / \mathrm{s}]$ \\
\hline SIFS & $10[\mu s]$ \\
\hline DIFS & $50[\mu s]$ \\
\hline CTS transmission time & $202[\mu s]$ \\
\hline RTS transmission time & $207[\mu s]$ \\
\hline ACK transmission time & $28[\mu s]$ \\
\hline Slot time & $20[\mu s]$ \\
\hline Maximum CW & 1023 \\
\hline Minimum CW & 15 \\
\hline
\end{tabular}




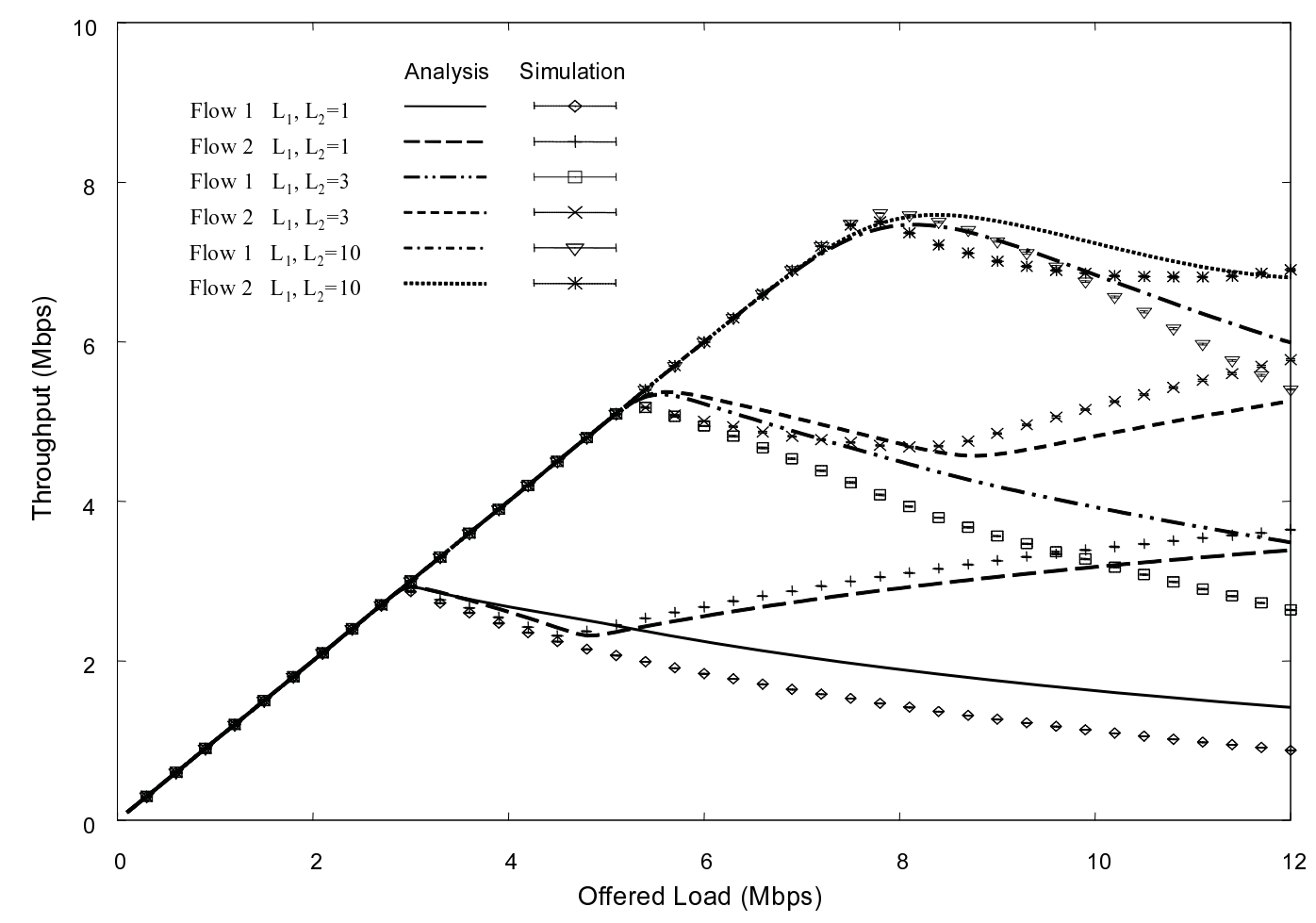

Figure 3: Comparison of analysis and simulation: E2ETs of Flows 1 and 2 vs. offered load. 


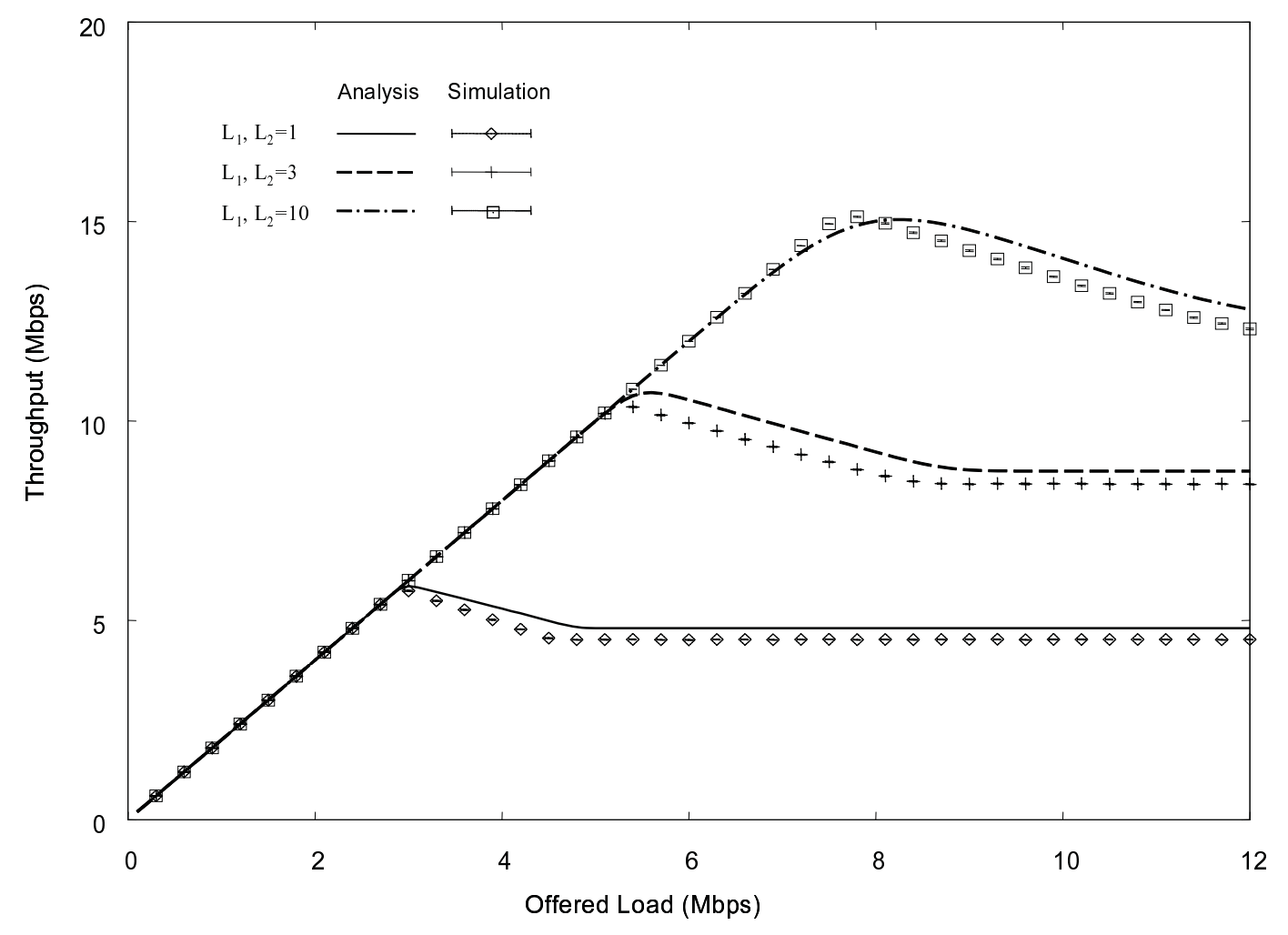

Figure 4: Comparison of analysis and simulation: overall throughput vs. offered load. 


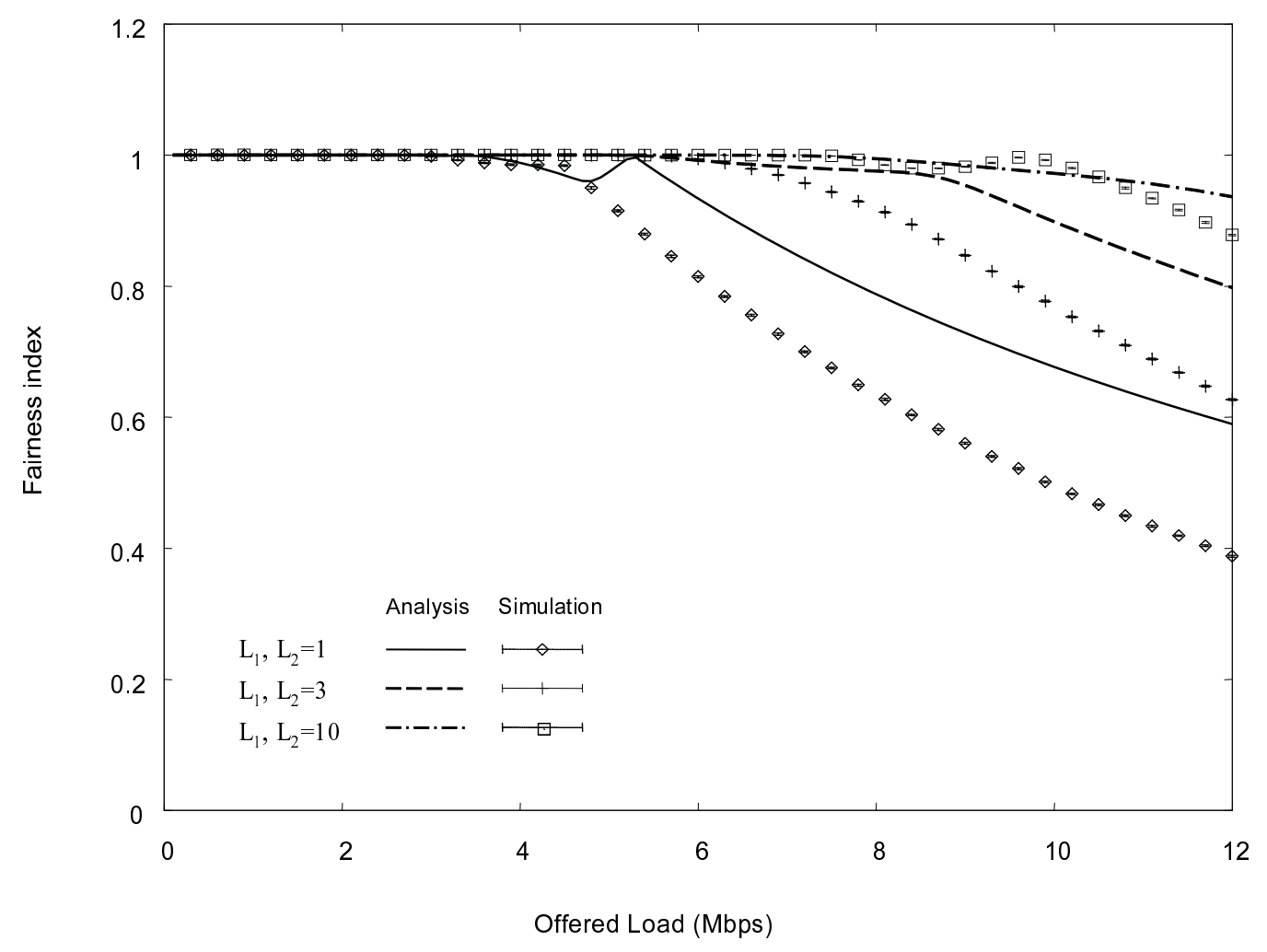

Figure 5: Fairness index vs. offered load. 


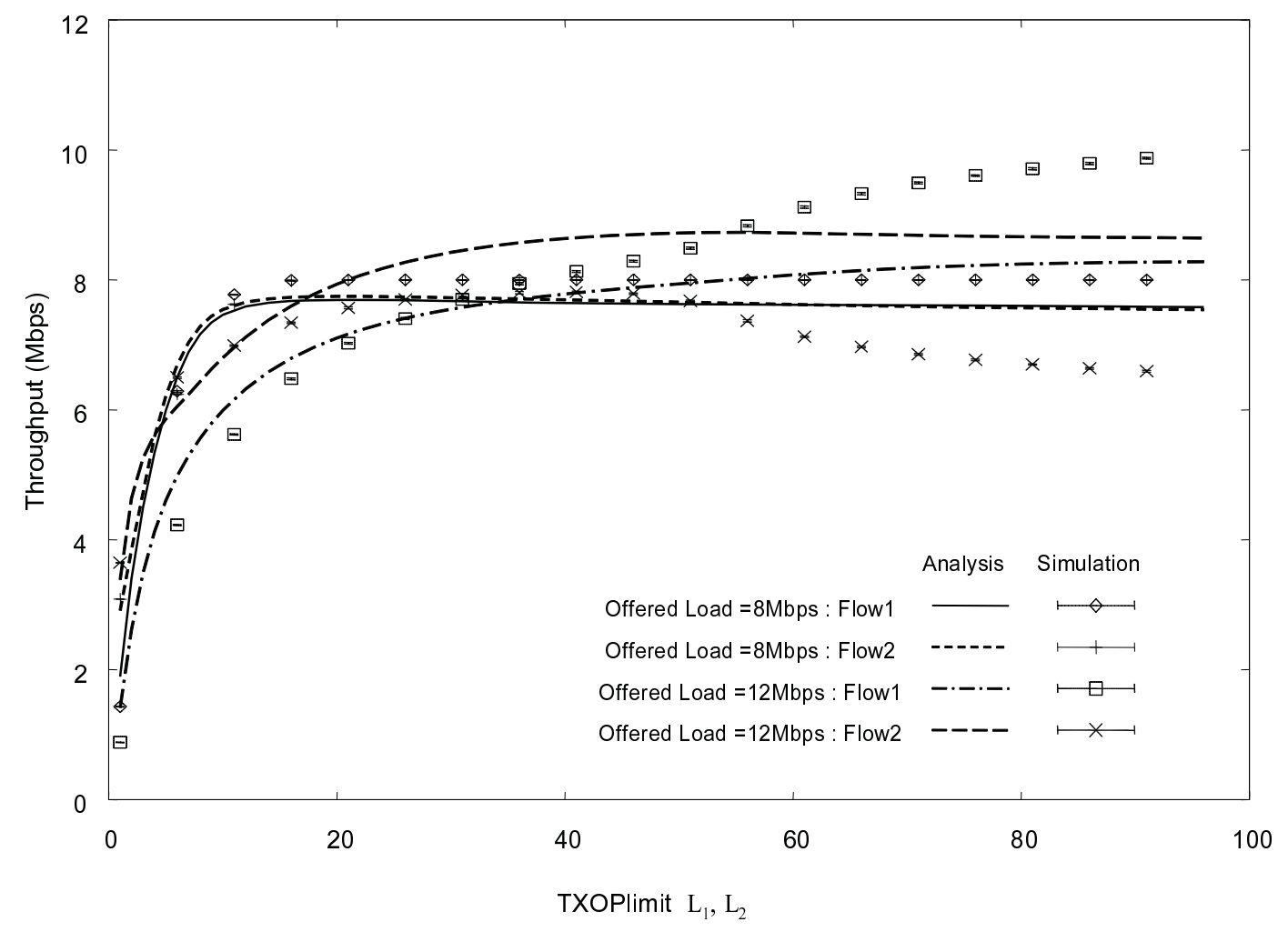

Figure 6: E2ETs of Flows 1 and 2 vs. TXOPlimit. 


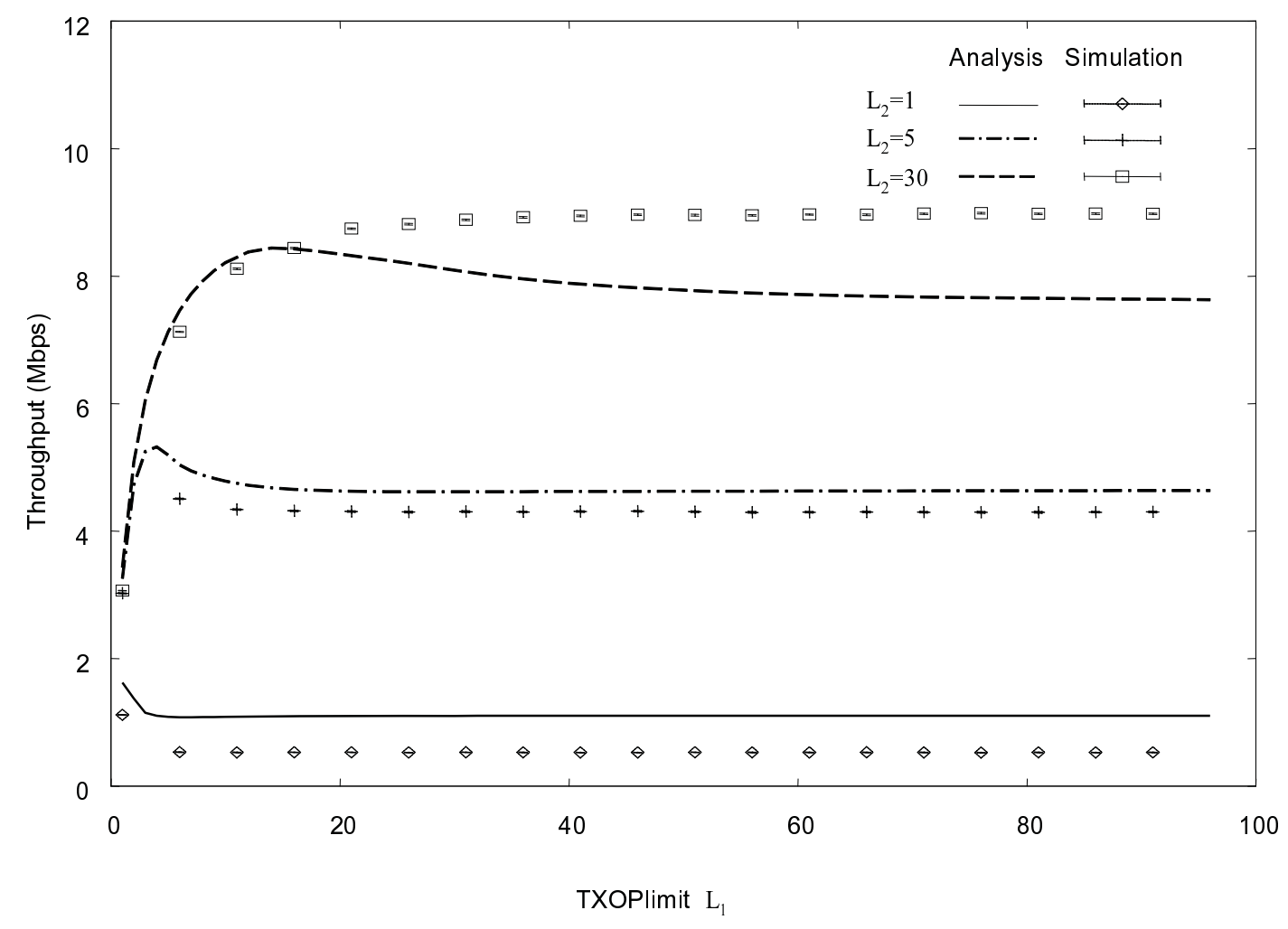

Figure 7: E2ET of Flow 1 vs $L_{1}$. (Offered load of Flows 1 and $\left.2=10[\mathrm{Mb} / \mathrm{s}]\right)$ 


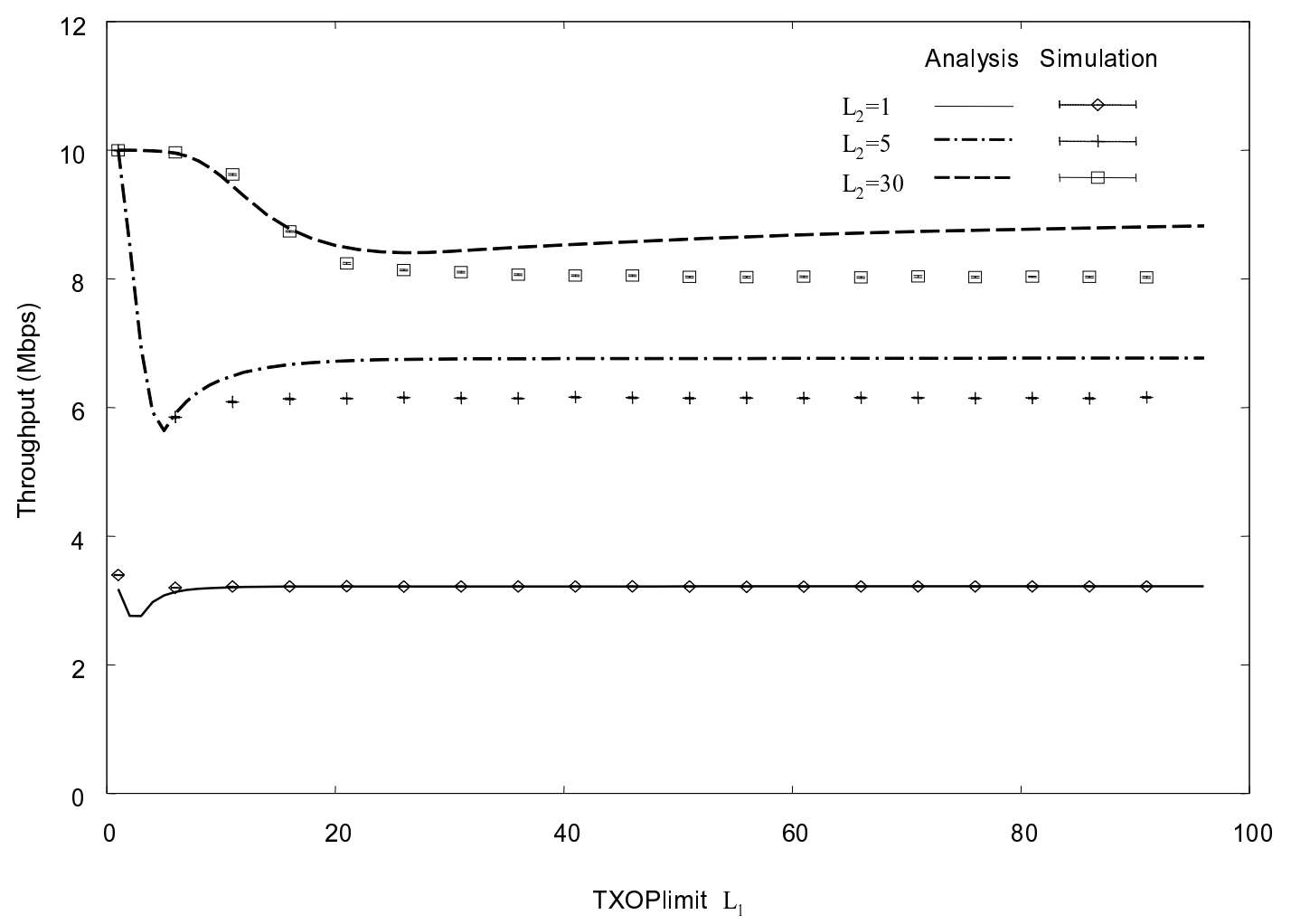

Figure 8: E2ET of Flow 2 vs. $L_{1}$. (Offered load of Flows 1 and $2=10[\mathrm{Mb} / \mathrm{s}]$ ) 


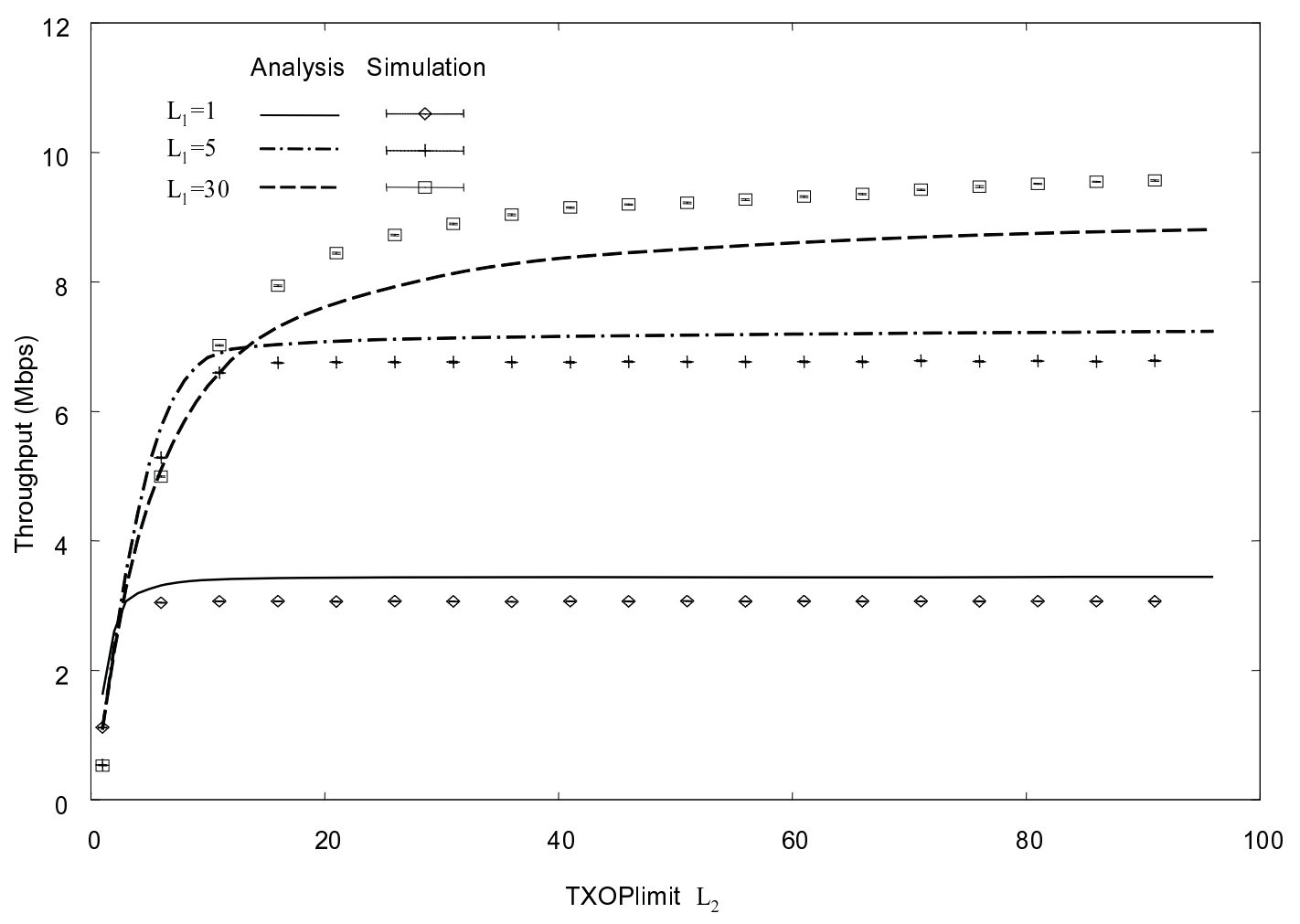

Figure 9: E2ET of Flow 1 vs. $L_{2}$. (Offered load of Flows 1 and $2=10[\mathrm{Mb} / \mathrm{s}]$ ) 


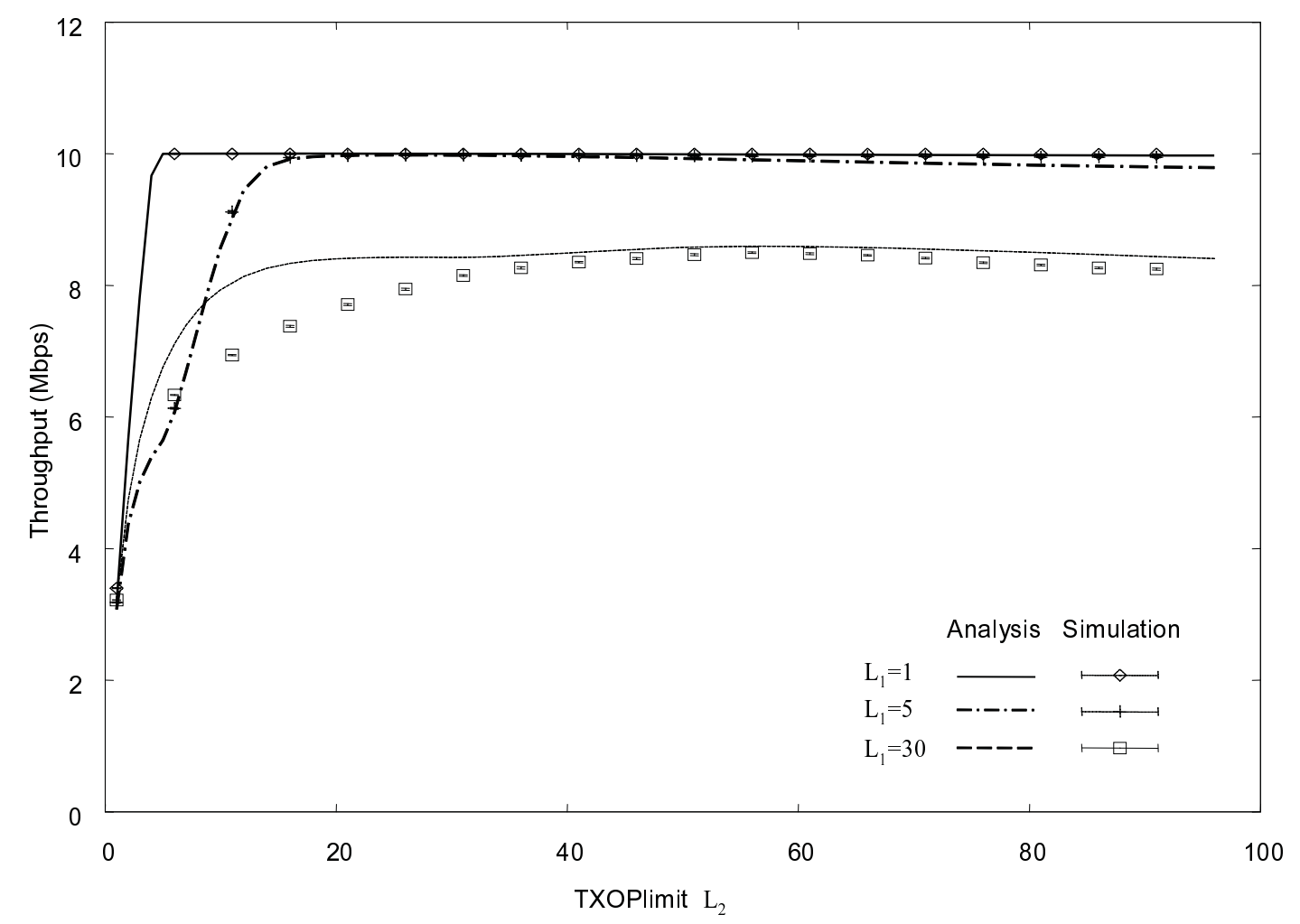

Figure 10: E2ET of Flow 2 vs. $L_{2}$. (Offered load of Flows 1 and $2=10[\mathrm{Mb} / \mathrm{s}]$ ) 